\title{
Elongation Prediction of Strip Steel in Annealing Furnace based on KPCA and Optimized LSSVM with ICPSO
}

\author{
C. Wang, J.H. Wang, S.S. Gu, X.K. Fang \\ College of Information Science and Engineering, \\ Northeastern University \\ China
}

\begin{abstract}
The elongation prediction of strips in furnace is extremely important in annealing process, which determines the quality and yield of product [1, 2]. Furthermore, the safety of airknife also depends on the prediction accuracy $[3,4]$. Thus, the optimal soft-sensing method is proposed based on kernel principal component analysis (KPCA) and optimized weighted least squares support vector machine (WLSSVM) by immune clone particle swarm optimization (ICPSO). Avoiding the particles are easy to sink into premature convergence and run into local optimization in the iterative process by using ICPSO , which generated by particle swarm optimization (PSO) algorithm, and the ICPSO is also used to optimize the parameters of WLSSVM. Then, the method uses KPCA to denoise the input data set and capture the high-dimensional nonlinear principal components in input data space, and the principal components are input into the ICPSO-WLSSVM model to establish the softsensing prediction model. The proposed method is successfully applied in the strip elongation prediction in annealing furnace. The simulations show that the KPCA and ICPSO-WLSSVM model has higher prediction accuracy compared with other algorithms that verified with production data.
\end{abstract}

Keywords-kernel principal component analysis; strip elongation; immune clone particle swarm optimization; least squares support vector machine; soft-sensing

\section{INTRODUCTION}

In the process of continuous annealing, the dual physical and chemical changes can make the strips extend or shorten, which passed through the preheating section (PHS), radiation heating section (HS), slow cooling section (SS), rapid cooling section (RCS) and other temperature sections with the tension action of rollers in the furnace, the process is shown in fig.1. Because of the space between galvanized section (POT) and annealing furnace outlet can hardly be installed any sensor to detect the elongation and welding seam position of strips, the soft-sensing technique of prediction with the offline and online information obtained by the sensors of temperature, tension, speed and etc. to enhance the production yield and the safety of air-knife is necessary.

\author{
Y.X. Zhang \\ School of Electrical Engineering, Shenyang University of \\ Technology \\ China
}

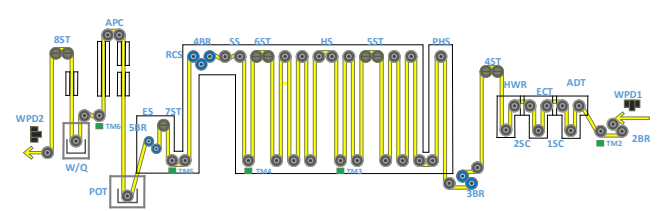

FIGURE I. GENERAL SCHEME OF STRIP STEEL IN ANNEALING PROCESS.

In this paper, a soft-sensing approach based on Kernel principal component analysis (KPCA) and hybrid novel particle swarm optimization (ICPSO) algorithm and weighted LSSVM (WLSSVM) is proposed to predict the strip elongation. The performance of basic PSO greatly depends on its parameters and it often suffers from being trapped in local optima which leads to premature convergence. Thus, immune clone algorithm (ICA) is further presented to enhance the diversity of population particles and the reliability of global convergence, and overcome the parameter sensitivity issue while efficiently controlling the global search of WLSSVM model with immune clonal selection strategy as well. To avoid solving the eigenvectors in the whole feature space, the softsensing method extracts features using KPCA, and trains the ICPSO-WLSSVM model with feature information extracted to obtain the optimal prediction model. Compared with other prediction methods, the soft-sensing approach proposed in this paper shows the higher prediction precision.

\section{KERNEL PRINCIPAL COMPONENT ANALYSIS}

This algorithm was originally proposed by Schölkopf et al [5]. The idea of KPCA is to firstly map the original input vectors $x_{i}$ into a high-dimensional feature space $\phi(x)$, which has been centralized $\sum_{k=1}^{M} \phi(x)=0$ in the feature space $F$, and the $M$ is the number of training samples, the corresponding

covariance matrix can be defined as:

$$
C=\frac{1}{M} \sum_{i=1}^{M} \phi\left(x_{i}\right) \cdot \phi\left(x_{i}\right)^{T}
$$

The centred kernel matrix $\tilde{K}$ can be defined as: 


$$
\tilde{K}_{i j}=\left(K-A_{M} K-K A_{M}+A_{M} K A_{M}\right)_{i j}
$$

where $A_{M}$ is a $M \times M$ matrix in which all the elements are equal to $1 / M$.Then the $n$th kernel principal component (KPC) representation is readily obtained:

$$
V^{n} \phi\left(x_{i}\right)=\sum_{v=1}^{M} \alpha_{v}^{n} K\left(x_{v}, x_{i}\right), i=1,2, \ldots, M
$$

\section{The Soft-Sensing Model FOR PREDICTION}

\section{A. ICPSO Algorithm}

In the PSO algorithm, every particle updates itself by tailing after the best

individual and global locations in each iteration [6, 7]:

$$
\begin{gathered}
v_{k+1}^{i}=w_{k} v_{k}^{i}+c_{1} r_{1}\left(\text { pbest }-x_{k}^{i}\right)+c_{2} r_{2}\left(\text { gbest }-x_{k}^{i}\right) \\
x_{k+1}^{i}=x_{k}^{i}+v_{k+1}^{i}
\end{gathered}
$$

where $w_{k}$ is called the inertia weight which controls the speed of next iteration [8]. $c_{1}$ and $c_{2}$ are positive constants, called acceleration coefficients. $r_{1}$ and $r_{2}$ are uniformly distributed random numbers generated in the range of $[0,1]$, and $k$ is the iteration counter. iter $_{\max }$ is the maximal number of iteration. The ICPSO algorithm can enhance the diversity in a colony and the reliability of global convergence, and it has the desirable characteristics in optimization and offers significant advantages over the simplex PSO algorithm [9].

(1) Clone Operation: Perform the clone operator $\Theta$ on the population and define $n_{c}$ as a given value relating to the clone scale, an antibody $Q=x^{1}, x^{2}, x^{3} \ldots x^{D}$ represents the local best position $p_{\text {best }}$ at one iteration. The cloning operation is describe as follows [10, 11]:

$$
\begin{gathered}
\Theta(Q)=\Theta\left(\left\{x^{1}, x^{2}, \ldots, x^{D}\right\}\right)=\left[\Theta\left(Q_{1}\right), \Theta\left(Q_{2}\right), \ldots, \Theta\left(Q_{q_{i}}\right)\right] \\
q_{i}=\operatorname{Int}\left(n_{c} \times \text { Popsize } / i, i=1,2, \ldots, \text { Popsize }\right)
\end{gathered}
$$

where $\operatorname{Int}(\cdot)$ denotes taking the integer value, Popsize is the size of the particles, the excellent antibodies are reproduced using fixed scale $n_{c}$.

(2) Immune Genic Operator: Perform the mutation operation as the immune genic operator. A new antibody population is obtained by mutation operation. It is defined as follows:

$$
\begin{gathered}
\operatorname{mutate}(Q)=\text { mutate }\left(\left\{x^{1}, \ldots, x^{D}\right\}\right)=\left\{x^{1}, x^{i^{\prime}}, \ldots, x^{D}\right\} \\
\xi_{1} \square=\pi \beta \varepsilon \sigma \tau+\rho \alpha \vee \delta
\end{gathered}
$$

the value of antibody's $i$ dimension changes in the operation of mutate, and we randomly generated randn in the range of $(0,1)$ [12].

(3) Recombination: Recombination can avoid the shortcoming that conventional recombination does not work when two antibodies are the same. It allows more antibodies information to be involved in the recombination.

(4) Fitness evaluation: The fitness indicates the error of prediction based on ICPSO, and a particle with lower the fitness shows that the particle can be selected to next generation by its excellent search capability. The function of fitness is

$$
\text { fitness }=\left(y_{i}^{*}-\hat{y}_{i}\right)^{2} / 2
$$

(5) Clone selection: The operation selects excellent particles after cloning to form new population into the next $\begin{array}{llllllllll}\mathrm{g} & \mathrm{e} & \mathrm{n} & \mathrm{e} & \mathrm{r} & \mathrm{a} & \mathrm{t} & \mathrm{i} & \mathrm{o} & \mathrm{n}\end{array}$

\section{B. Weighted LSSVM Algorithm}

The weighted least squares support vector machines (WLS-SVM) was introduced by Suykens et al [13] to decrease the training computational effort of SVM in large-scale problems and obtain a robust estimate.

Given a training set of $\mathrm{N}$ data points $\left\{y_{k}, \mathrm{x}_{k}\right\}_{k=1}^{N}$, where $x_{k} \in R^{n}$ is the $k$ th input pattern and $v_{k} \in R$ is the $k$ th output pattern, the model of WLSSVM is defined as follows:

$$
L(w, b, e ; \alpha)=\frac{1}{2} w^{T} w+\frac{\gamma}{2} v_{k} \sum_{k=1}^{N} e_{k}^{2}-\sum_{k=1}^{N} \alpha_{k}\left\{y_{k}\left[w^{T} \phi(x)+b\right]-1+e_{k}\right\}
$$

where $\alpha_{k}(i=1,2, \ldots, N)$ are the Lagrange multipliers, $v_{k}$ is the weighted factor.

The radial basis function (RBF) kernel is applied because it is capable to nonlinearly map the training data into an infinite dimensional space, thus, it is suitable to deal with nonlinear relationship problems. $\sigma^{2}$ is a positive real constant, and it is usually called the RBF kernel width. The regression function of WLSSVM can be given by

$$
y(x)=\operatorname{sign}\left[\sum_{k=1}^{N} \alpha_{k} y_{k} K\left(x, x_{k}\right)+b\right]
$$

\section{Hybrid ICPSO-WLSSVM Alg\$orithm}

In this paper, the ICPSO algorithm are proposed to optimize parameters to verify the objective of the problem. The executive steps of the ICPSO are as follows:

Step 1: Initialize particles and set ICPSO parameters. Set the ICPSO parameters including the population size $N$, maximum number of iterations iter $_{\max }$, fitness of each particle according to eqn (9), weighted factor $w_{k}$, and the range of the 
velocity between $-v_{\max }$ to $v_{\max }$, where $v_{\max }$ is a predefined boundary value

according to the corresponding experiment data;

Step 2: Evaluate the fitness of particle according to eqn (9), and set the $p_{\text {best }}$ from the particle with the minimal fitness in the swarm. From the $p_{\text {best }}$ particles, we can get the initial $g_{b e s t}$;

Step 3: Update the velocity and position of the particle according to eqns $(4,5)$. Update the individual best position to obtain the global best position $p_{\text {best }}$

according to the fitness calculation results;

Step 4: Made $m$ clones of the $p_{\text {best }}$ according to eqns $(6,7)$, mutate the particles

according to eqns $(8,9)$;

Step 5: According to the prediction of the model WLSSVM, calculate the fitness of particles which are generated by cloning and mutating. Clone the particles

which have the better fitness to form a new population;

Step 6: Update the $g_{\text {best }}$ using the current $p_{\text {best }}$;

Step 7: Set the iterative variable $i=i+1$, terminate the algorithm if criterion $\left(i>\right.$ iter $\left._{\max }\right)$ is satisfied, and take the updated $g_{\text {bes }} t$ as the optimal hyper-parameters of

the model WLSSVM, else go back to Step 4.

\section{Case Study On ElOngation Prediction OF Strips}

In this section, the proposed method is applied in the annealing of strips process.

A training data set including 3000 normal samples is used to build the prediction models. The testing data is composed of 1000 samples. The initial parameters of the PSO algorithm, the dimension number $D=2$, the particle number Popsize $N=30$, the positive constants $c_{1}$ and $c_{2}$ are initialized to 2 . The modified $v_{\max }$ is set to 2 , iter $\max =200$. The inertia weight $w_{k}$ concluding $w_{\max }=0.8$ and $w_{\min }=0.2$. In the IC algorithm, the parameter $s=1$. In the WLSSVM algorithm, RBF is chosen as kernel function with the parameter $\sigma^{2}=0.1$, the distribution parameters $\alpha=0.3$.

fig. $2(a-c)$ show the detailed description of deviations between the measured and predicted values of elongation of strips on the training data set with three different models. It is clear that the KPCA-ICPSO-WLSSVM model exhibits the better approximation accuracy and the prediction deviations vary within a small range around $[-1,1]$. The comparison between elongation deviation of strips in the annealing furnace and prediction with approach proposed on practical testing data set is showed in fig. $2(d)$. It can be found that the KPCAICPSO-WLSSVM model have good prediction accuracy on testing data set, because the prediction errors are obviously less than the values estimated by current method used in the practical engineering.

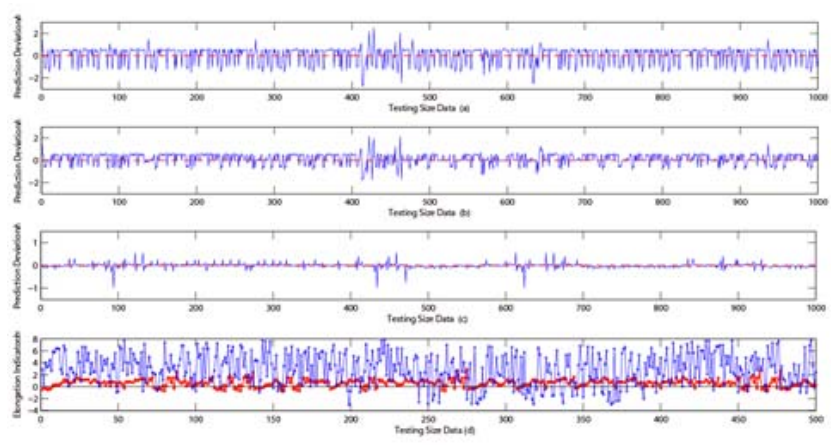

FIGURE II. PREDICTION DEVIATIONS OF ELONGATION OF DIFFERENT MODELS: (A) PSO-LSSVM, (B) ICPSO-LSSVM, (C) KPCAICPSO-WLSSVM, (D)COMPARED WITH CURRENT METHOD.

The detailed comparison of test performance with PSOLSSVM, ICPSO- LSSVM and KPCA-ICPSO-WLSSVM is listed in Table I, and the difference between the output of the models and the real output desired output is considered as the error and represented in several methods, including mean absolute error (MAE), mean relative error (MRE), root mean square error (RMSE), standard

deviation of absolute error (STD), and Theil's Inequality Coefficient (TIC).

The digital comparison in Table 1 indicates that performance of the algorithm proposed is the best on overall. The MAE, MRE and RMSE listed also in Table I have confirmed the prediction accuracy of the proposed method. KPCA-ICPSO -LSSVM has the smallest STD among the three models, which indicates the predictive stability of the method. Similar results are observed in terms of TIC, which indicates a good level of agreement between the proposed model and the

studied process.

TABLE I. COMPARISON OF DIFFERENT MODELS

\begin{tabular}{cccccc}
\hline Model & MAE & MRE (\%) & RMSE & STD & TIC \\
\hline PSO-LSSVM & 0.6148 & 5.082 & 0.1076 & 0.1213 & 0.9101 \\
ICPSO-LSSVM & 0.4742 & 3.673 & 0.1132 & 0.1073 & 0.0731 \\
$\begin{array}{c}\text { KPCA-ICPSO- } \\
\text { WLSSVM }\end{array}$ & 0.0502 & 1.732 & 0.0823 & 0.0732 & 0.0232 \\
\hline
\end{tabular}

\section{CONCLUSION}

The prediction of the elongation of strip steel in annealing furnace using KPCA-ICPSO-WLSSVM model, which determined the quality and yield of product, is presented in this paper. To overcome the premature convergence of PSO algorithm, the ICPSO is represented. To further reduce the deviations in the prediction, the WLSSVM is used to obtain robust estimates for regression. In order to deal with nonlinear characteristics of data set, kernel principal component analysis (KPCA) is applied to extract and reduce features. For comparison, PSO-LSSVM, ICPSO-LSSVM, ICPSOWLSSVM and KPCA-ICPSO-WLSSVM are developed and evaluated. The application of the proposed method to the test and data obtained from the industrial plant demonstrates its effectiveness and reliability. The KPCA-ICPSO-WLSSVM 
model predicts the elongation with MRE of $1.732 \%$ on the test data set, which is much more accurate than other models. For these reasons, the KPCA-ICPSO-WLSSVM model proposed can be accepted as very successfully for elongation prediction of strips in annealing process.

\section{ACKNOWLEDGMENTS}

This Project was supported by the National Natural Science Foundation of China (Grant No.61102124), and Liaoning Key Industry Programme (Grant No.2010220014).

\section{REFERENCES}

[1] R. BAI, Z.S. ZHANG, T.Y. CHAI. Modeling and Simulation for Strip Tension in Continuous Annealing Process [J]. Journal of System Simulation, 2007, 19(23): 5477-5481.

[2] J.H. Wang, C. Wang, X.F. Zhu, X.K. Fang. Application of Soft Sensor in Welding Seam Tracking Prediction Based on LSSVM and PSO with Compression Factor [C]. 2013 25th Chinese Control and Decision Conference, 2013, 2441-2446.

[3] S.Y. Tian, R.S. Shi, X.H. Zhu, Q.H. Yang, L.H. Chen. Application of ultrasonic sensor to broken line tracking of corrugated board [J]. TRANSACTIONS OF THE CH INAWELDING INSTITUTION, 2010, 31(12): 97-100.

[4] X.D. Gao, X.G. Zhong, D.Y. You, Seiji Katayama. Kalman Filtering Compensated by Radial Basis Function Neural Network for Seam Tracking of Laser Welding [J]. IEEE TRANSACTIONS ON CONTROL SYSTEMS TECHNOLOGY, 2013, 21(5): 1916-1923.

[5] B. Schölkopf., A. Somola, K. Müller, Nonliear component analysis as a kernel eigenvalue problem, Neural Comput. 10 (1998) 1299-1319.

[6] James Kennedy, Russell C. Eberhart. Particle swarm optimization, Proc of IEEE International Conference on Networks. Piscataway: IEEE Press, pp.1942-1948, 1995.

[7] J. Kennedy, R. Eberhart, Particle swarm optimization, IEEE Int. Conf. Neural Networks (1995) 1942-1948.

[8] F.M. Burnet. The Clonal Selection Theory of Acquired Immunity, Cambridge University Press, London, 1959.

[9] H.Q. Jiang, Z.B. Yan, X.G. Liu. Melt index prediction using optimized least squares support vector machines based on hybrid particle swarm optimization algorithm [J]. Neurocomputing , 2013, 119 (2013): 469-477.

[10] M.G. Gong, H.F. Du, L.C. Jiao. Optimal approximation of linear systems by artificial immune response [J]. Science in China, 2006, 49 (1), 63-79.

[11] Shuiping Gou n, Xiong Zhuang, Yangyang Li, Cong Xu, Licheng C. Jiao. Multi-elitist immune clonal quantum clustering algorithm [J].Neurocomputing, 2013, 101 (2013): 275-289.

[12] H. Modares, A. Alfi, S. Naghibi, B. Mohammad. Parameter estimation of bilinear systems based on an adaptive particle swarm optimization [J]. Engineering Applications of Artificial Intelligence. 2010, 23 (7): 11051111.

[13] Suykens JAK, Brabanter JD, Lukas L, Vandewalle J. Weighted least squares support vector machines: robustness and sparse approximation. Neurocomputing, 2002, 48 (2002): 85-105. 